\title{
Outage probability of a relay strategy allowing intra-link errors utilizing Slepian-Wolf theorem
}

\author{
Meng Cheng ${ }^{1 *}$, Khoirul Anwar $^{1}$ and Tad Matsumoto ${ }^{1,2}$
}

\begin{abstract}
In conventional decode-and-forward (DF) one-way relay systems, a data block received at the relay node is discarded, if the information part is found to have errors after decoding. Such errors are referred to as intra-link errors in this article. However, in a setup where the relay forwards data blocks despite possible intra-link errors, the two data blocks, one from the source node and the other from the relay node, are highly correlated because they were transmitted from the same source. In this article, we focus on the outage probability analysis of such a relay transmission system, where source-destination and relay-destination links, Link 1 and Link 2, respectively, are assumed to suffer from the correlated fading variation due to block Rayleigh fading. The intra-link is assumed to be represented by a simple bit-flipping model, where some of the information bits recovered at the relay node are the flipped version of their corresponding original information bits at the source. The correlated bit streams are encoded separately by the source and relay nodes, and transmitted block-by-block to a common destination using different time slots, where the information sequence transmitted over Link 2 may be a noise-corrupted interleaved version of the original sequence. The joint decoding takes place at the destination by exploiting the correlation knowledge of the intra-link (source-relay link). It is shown that the outage probability of the proposed transmission technique can be expressed by a set of double integrals over the admissible rate range, given by the Slepian-Wolf theorem, with respect to the probability density function ( $p d f$ ) of the instantaneous signal-to-noise power ratios (SNR) of Link 1 and Link 2. It is found that, with the Slepian-Wolf relay technique, so far as the correlation $\rho$ of the complex fading variation is $|\rho|<1$, the 2 nd order diversity can be achieved only if the two bit streams are fully correlated. This indicates that the diversity order exhibited in the outage curve converges to 1 when the bit streams are not fully correlated. Moreover, the Slepian-Wolf outage probability is proved to be smaller than that of the 2 nd order maximum ratio combining (MRC) diversity, if the average SNRs of the two independent links are the same. Exact as well as asymptotic expressions of the outage probability are theoretically derived in the article. In addition, the theoretical outage results are compared with the frame-error-rate (FER) curves, obtained by a series of simulations for the Slepian-Wolf relay system based on bit-interleaved coded modulation with iterative detection (BICM-ID). It is shown that the FER curves exhibit the same tendency as the theoretical results.
\end{abstract}

\section{Introduction}

In many wireless communication systems, channels are assumed to suffer from variations due to fading. One of the most reasonable and hence widely accepted model for block-wise transmission is the block Rayleigh fading channel, where the channel realization changes block-byblock. With the block fading assumption, if the channel state information (CSI) is unknown to the transmitter,

\footnotetext{
*Correspondence: chengmeng@jaist.ac.jp

1 School of Information Science, Japan Advanced Institute of Science and

Technology (JAIST), 1-1 Asahidai, Nomi, Ishikawa 923-1292, Japan

Full list of author information is available at the end of the article
}

achieving always error-free transmission is not possible. This is simply because the transmission chain parameter cannot be flexibly changed at the transmitter without the knowledge of CSI, which invokes the necessity of the outage analysis. The result of the outage analysis provides the system operators estimate with which probability the quality of service (QoS) requirement can be satisfied [1].

Relay systems [2] have been well studied in the recent years, assuming various relay strategies, such as amplifyand-forward (AF) and decode-and-forward (DF) techniques $[3,4]$, etc. In the case of DF, the information bit streams recovered at the relay may contain errors

\section{是 Springer}

C) 2013 Cheng et al: licensee Springer. This is an Open Access article distributed under the terms of the Creative Commons Attribution License (http://creativecommons.org/licenses/by/2.0), which permits unrestricted use, distribution, and reproduction in any medium, provided the original work is properly cited. 
occurring in the source-relay link, referred to as the intralink in this article. In conventional DF systems, the recovered data block is discarded if the relay detects errors in the information part after decoding. However, in a setup where the relay forwards data blocks despite possible intra-link errors, the information streams received by the destination via the source-destination link (Link 1 ) and the relay-destination (Link 2) are highly correlated, because they were transmitted from the same source. In [5], the idea of the Slepian-Wolf coding is first introduced in wireless cooperation scenarios with the aim of exploiting the distributed correlated source coding concept. Reference [6] also formulates the issue from the viewpoint of the Slepian-Wolf theorem, in a different way from [5], where the intra-link error probability is used to express the source-relay correlation [6]. The intra-link error probability can be estimated and further utilized in the joint decoding process at the destination node, by utilizing a log-likelihood ratio (LLR) updating function [6].

The primary goal of this article is to theoretically analyze the outage probability of the relay system presented in [6] and its asymptotic properties, both based on the Slepian-Wolf theorem. Originally, the Slepian-Wolf theorem determines the admissible rate region for the compression of correlated sources. The theorem states that compared with the case where the information bit streams are assumed to be independent, higher compression rate can be achieved by exploiting the correlation knowledge of the sources. Furthermore, the two correlated source streams can be separately encoded at each transmitter side, and the compressed data received by the receiver are jointly decoded by a common decoder. According to the Slepian-Wolf theorem, by utilizing the correlation knowledge of the sources at the receiver, the joint decoder can achieve the same compression rate as in the case that the sources are encoded by optimum joint encoders. This theorem can be applied to many practical applications, such as the sensor networks where measurements of the same object are performed by the separated sensors, and hence, the results are correlated.

This article assumes a bit-flipping model for the intralink transmission [7], where some of the recovered bits $b_{2}$ at the relay node are flipped versions of their corresponding original information bits $b_{1}$ at the source node. Hence, $b_{2}=b_{1} \oplus e$ with probability $\operatorname{Pr}(e=1)=p_{e}$, and since $\mu=\left\langle\left(2 b_{1}-1\right)\left(2 b_{2}-1\right)\right\rangle=1-2 p_{e},(-1 \leq \mu \leq 1)$, with $\langle\cdot\rangle$ indicating the expectation operator, we use $p_{e}$ and $\mu$ both equivalently describing the correlation between $b_{1}$ and $b_{2}$ [8]. In fact, the value of $p_{e}$ depends on many parameters, related to the intra-link transmission such as modulation-and-detection schemes and/or encodingand-decoding methods. However, this article uses $p_{e}$ as a parameter. The $p_{e}$ value can be estimated by the destination, block-by-block, as presented by [6], and hence, this assumption is reasonable in practical applications. On the other hand, if we would calculate the outage probability of the system where $p_{e}$ is assumed to be a random variable, we would have to derive the distribution of $p_{e}$, which is not practical due to the fact described above. Hence, we assume that the intra-link is stable and hence $p_{e}$ is a constant. The scenario under consideration is exemplified as depicted by Figure 1. In the assumed scenario, the channel gains of either one or both of the two links fade below the threshold values required for successful transmission, which defines the outage event. In this study, we show that the theoretical outage probability of the Slepian-Wolf relay system can be expressed by double integrals over the admissible rate region defined by the Slepian-Wolf theorem, with regard to the probability density function $(p d f)$ of the instantaneous signal-to-noise power ratios (SNRs).

This article is organized as follows. The relay transmission model assumed in this article is introduced in Section 2. The admissible rate region is defined in Section 3, based on the Slepian-Wolf theorem, for the relay transmission model introduced in Section 2. The outage probabilities of the Slepian-Wolf relay system are then derived theoretically in Section 3, assuming that Link 1 and Link 2 are suffering from correlated block Rayleigh fading, where the fading variation of Link 1 and Link 2 are assumed to be correlated because of the scenarios assumption presented in Figure 1. The theoretical proofs of the asymptotic tendency of the outage curves are presented in Section 4. In Section 5, we apply the SlepianWolf relay technique provided in [6] to a bit-interleaved

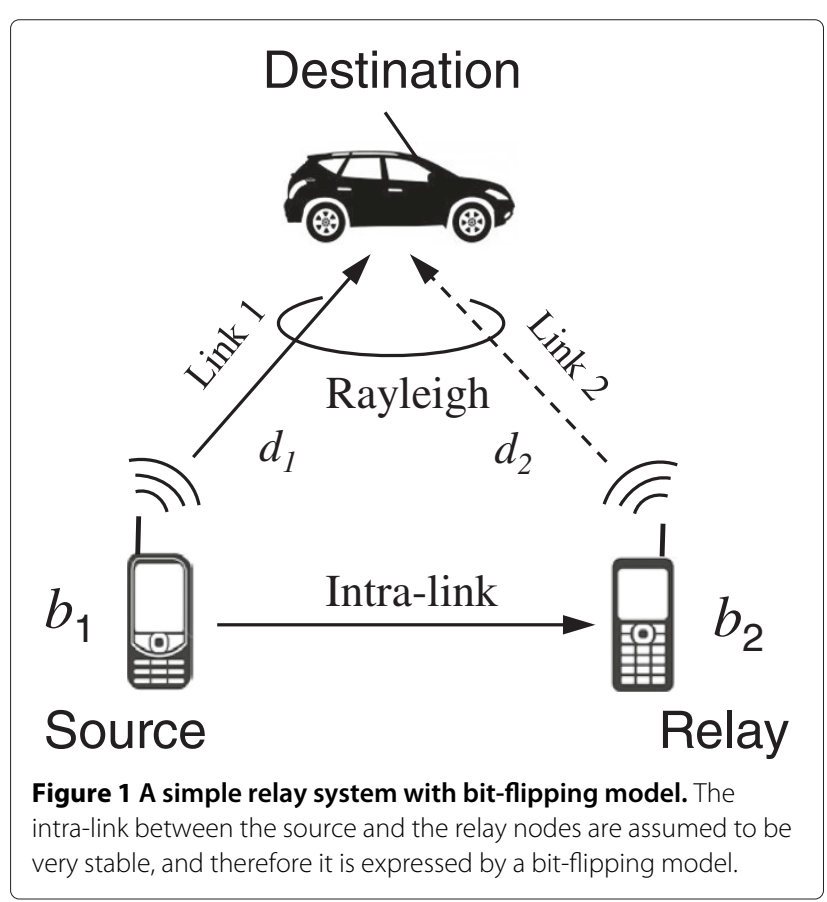


coded modulation with iterative detection (BICM-ID), as an example, to demonstrate how the correlation knowledge can be used. Numerical results are presented in Section 6, where it is verified that the performance tendency obtained by Section 5 is consistent to the simulated results. Conclusions are drawn in Section 7 with same concluding results.

\section{System model}

We consider a simple one-way relay transmission model, as shown in Figure 1. The original information bit stream $b_{1}$ is broadcasted from the source node during the first time slot. The relay aims to recover $b_{1}$ before re-encoding and forwarding it to the destination during the second time slot, as in the DF strategy. The recovery of $b_{1}$ might not be perfect, however, the proposed Slepian-Wolf relaying system will allow re-transmission despite possible intra-link errors.

Because of the reason described in the introduction, the intra-link error probability $p_{e}$ is assumed to be constant, even though $p_{e}$ can be estimated by the destination only [6], block-by-block, in practice. $p_{e}$ does not have to represent the bit error probability of the real intra-link signal transmission. It represents the error probability of the virtual link between source and relay node: it may be before or after the decoding of the channel code. This article only uses the probability $p_{e}$ as a parameter in theoretical analyzes regardless of the intra-link transmission scheme.

With $d_{1}$ and $d_{2}$ representing the lengths of Link 1 and Link 2, respectively, and with the geometrical gain [9] of Link $1, G_{1}$, being normalized to 1 , the geometric gain of Link 2, relative to Link 1, can be defined as

$$
G_{2}=\left(\frac{d_{1}}{d_{2}}\right)^{\alpha},
$$

where in this article the path loss factor $\alpha$ is set at 3.52 as in [10]. The received signals $y_{1}$ and $y_{2}$ at the destination, received via Link 1 and Link 2, respectively, are expressed as follows:

$$
\begin{aligned}
& y_{1}=\sqrt{G_{1}} h_{1} s_{1}+n_{1}, \\
& y_{2}=\sqrt{G_{2}} h_{2} s_{2}+n_{2},
\end{aligned}
$$

where $s_{1}$ and $s_{2}$ are the modulated symbols transmitted from the source and relay nodes, respectively, and $n_{i}$ and $h_{i}(i=1,2)$ denote the zero-mean white additive Gaussian noises (AWGN) with the variance $\sigma_{i}^{2}$ per dimension and the complex channel gain of Link $i$, respectively. It is assumed that $\sigma_{1}^{2}=\sigma_{2}^{2}=\sigma^{2}$ and $h_{1}$ and $h_{2}$ are kept constant over one block duration due to the block Rayleigh fading assumption. With the definitions described above, the instantaneous SNR of Link $i$ is denoted by $\gamma_{i}=$ $G_{i}\left|h_{i}\right|^{2} E_{s, i} /\left(2 \sigma^{2}\right)$, where $E_{s, i}$ represents the per-symbol signal power of Link $i$. The joint decoding process then takes place for the received signals $y_{s d}$ and $y_{r d}$ at the destination node.

\section{Outage probability derivation}

\subsection{Outage definition}

The definition of the outage probability of the proposed Slepian-Wolf relay system is derived in this subsection. Let $R_{1}$ and $R_{2}$ denote the rates of the bit streams from the source and relay nodes, respectively. According to the Slepian-Wolf theorem [11], if $R_{1}$ and $R_{2}$ satisfy the following three inequalities, the transmitted data can be recovered with arbitrary low error probability.

$$
\begin{aligned}
& R_{1} \geqslant H\left(b_{1} \mid b_{2}\right), \\
& R_{2} \geqslant H\left(b_{2} \mid b_{1}\right), \\
& R_{1}+R_{2} \geqslant H\left(b_{1}, b_{2}\right),
\end{aligned}
$$

where $H\left(b_{1} \mid b_{2}\right)$ and $H\left(b_{2} \mid b_{1}\right)$ denote the conditional entropy of $b_{1}$ and $b_{2}$, given $b_{2}$ and $b_{1}$, respectively, and $H\left(b_{1}, b_{2}\right)$ denotes the joint entropy of the correlated information $b_{1}$ and $b_{2}$. When the rate $R_{1}$ for transmitting the information stream $b_{1}$ is equal to its entropy $H\left(b_{1}\right)$, the rate $R_{2}$ for transmitting the information stream $b_{2}$ can be less than its entropy $H\left(b_{2}\right)$, but it has to be larger than the conditional entropy $H\left(b_{2} \mid b_{1}\right)$, as indicated by the point $X_{1}$ in Figure 2. Similarly, when $b_{2}$ is transmitted at the rate $H\left(b_{2}\right)$, then $b_{1}$ can be transmitted at the rate which is less than $H\left(b_{1}\right)$ but should be larger than $H\left(b_{1} \mid b_{2}\right)$ as indicated by the point $X_{2}$ in Figure 2 . Since the binary symmetric source model $(\operatorname{Pr}(1)=\operatorname{Pr}(0)=0.5)$ is assumed in this article, $H\left(b_{1}\right)=H\left(b_{2}\right)=1, H\left(b_{1}\right.$ । $\left.b_{2}\right)=H\left(b_{2} \mid b_{1}\right)=H\left(p_{e}\right), H\left(b_{1}, b_{2}\right)=1+H\left(p_{e}\right)$ with

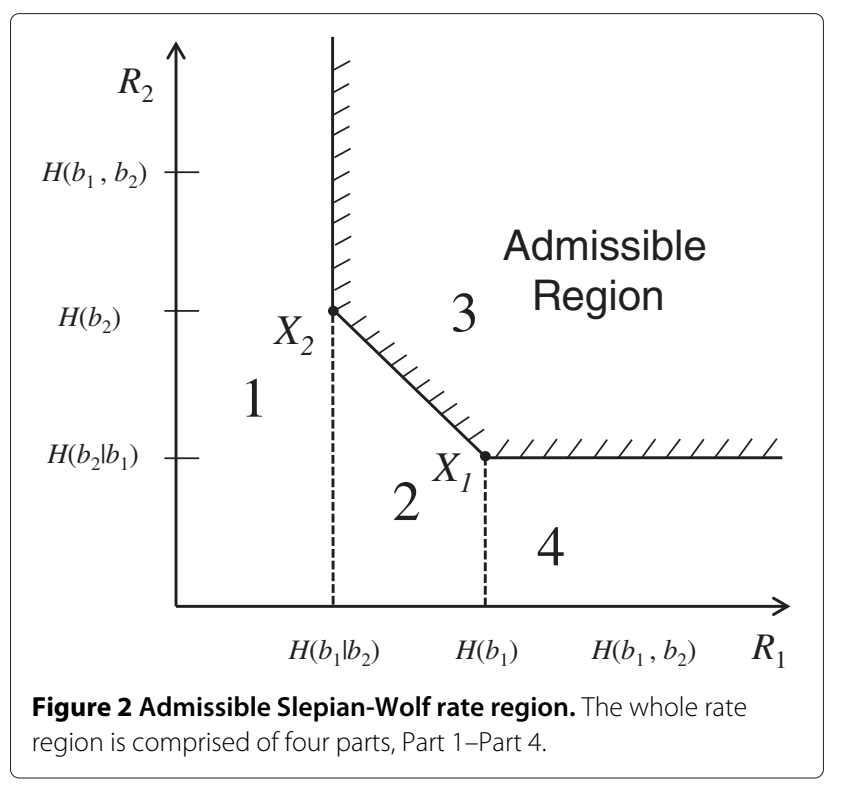


$H\left(p_{e}\right)=-p_{e} \log _{2}\left(p_{e}\right)-\left(1-p_{e}\right) \log _{2}\left(1-p_{e}\right)$. Assume that a channel capacity-achieving code is used for the transmission over Link $i$. The relationship between the threshold instantaneous SNR and its corresponding rate $R_{i}$ is given by

$$
R_{i}=\frac{1}{R_{c i}} \log \left(1+\gamma_{i}\right), \quad i=1,2
$$

where $R_{c i}$ represents the spectrum efficiency of the transmission chain, including the channel coding scheme and the modulation multiplicity within Link $i$ [7].

As shown in Figure 2, the entire rate region for the rate pair $R_{1}$ and $R_{2}$ can be divided into four parts, with $P_{j}, j=\{1,2,3,4\}$, representing the probability that $R_{1}$ and $R_{2}$ fall into Part $j$. The common admissible rate region for the case of two correlated sources can be expressed by an unbounded polygon, which corresponds to Part 3 of the rate region shown in Figure 2. The two correlated bit streams cannot be successfully recovered if the rates $R_{1}$ and $R_{2}$ are not within the admissible region Part 3 . Hence, the outage event happens when $R_{1}$ and $R_{2}$ fall outside the Slepian-Wolf admissible region, with the probability of

$$
\begin{aligned}
P_{\text {out }, \text { sw }} & =1-P_{3}, \\
& =P_{1}+P_{2}+P_{4} .
\end{aligned}
$$

However, in this article, both Part 3 and Part 4 are supposed to be included as the admissible rate region [12], because in the one-way Slepian-Wolf relay system investigated in this article, we only focus on the transmission of the source information $b_{1}$. The data to be transmitted from the relay node actually is the erroneous copy of the original information bit stream, interleaved by $\Pi_{0}$ as shown in Figure 3a. Therefore, analyzing the impact of Link 2 on the outage probability is our target. In other words, an arbitrary value of $R_{2}$ is satisfactory as long as $R_{1}$ is larger than $H\left(b_{1}\right)$. In this case, the individual outage event happens when the pair $\left(R_{1}, R_{2}\right)$ falls in Part 1 or Part 2, and the outage probability of the Slepian-Wolf relay model considered in this article is defined as

$$
\begin{aligned}
P_{\text {out }, \text { relay }} & =1-P_{3}-P_{4}, \\
& =P_{1}+P_{2} .
\end{aligned}
$$

Thus, the conditions on $R_{1}$ and $R_{2}$ to achieve arbitrary low bit error rate are given by ${ }^{\mathrm{a}}$

$$
\begin{aligned}
P_{1}= & \operatorname{Pr}\left[0<R_{1}<H\left(b_{1} \mid b_{2}\right), R_{2}>0\right], \\
= & \operatorname{Pr}\left[0<\gamma_{1}<2^{R_{c 1} H\left(b_{1} \mid b_{2}\right)}-1, \gamma_{2}>0\right] . \\
P_{2}= & \operatorname{Pr}\left[H\left(b_{1} \mid b_{2}\right)<R_{1}<H\left(b_{1}\right), R_{1}+R_{2}<H\left(b_{1}, b_{2}\right)\right], \\
= & \operatorname{Pr}\left[2^{R_{c 1} H\left(b_{1} \mid b_{2}\right)}-1<\gamma_{1}<2^{R_{c 1} H\left(b_{1}\right)}-1,\right. \\
& \left.\quad 0<\gamma_{2}<2^{\left[R_{c 2} H\left(b_{1}, b_{2}\right)-\frac{R_{c 2}}{R_{c 1}} \log \left(1+\gamma_{1}\right)\right]}-1\right] .
\end{aligned}
$$

\subsection{Outage calculation}

\subsubsection{Independent links}

In this section, the outage probability is derived, based on the Slepian-Wolf relay model. With an assumption that both Link 1 and Link 2 suffer from statistically independent block Rayleigh fading, the joint $p d f$ of the instantaneous SNR can be expressed as $p\left(\gamma_{1}, \gamma_{2}\right)=p\left(\gamma_{1}\right) p\left(\gamma_{2}\right)$, with [13]

$$
p\left(\gamma_{i}\right)=\frac{1}{\Gamma_{i}} \exp \left(-\frac{\gamma_{i}}{\Gamma_{i}}\right), \quad i=1,2
$$

where $\Gamma_{i}=G_{i} E_{s, i} /\left(2 \sigma^{2}\right)$, which represents the normalized average SNR of the $i$-th link. Based on (10) and (11), the probabilities $P_{1}$ and $P_{2}$ can be mathematically derived as follows

$$
\begin{aligned}
P_{1} & =\int_{\gamma_{1}=0}^{2^{R_{c 1} H\left(b_{1} \mid b_{2}\right)}-1} \int_{\gamma_{2}=0}^{\infty} p\left(\gamma_{1}\right) p\left(\gamma_{2}\right) d \gamma_{1} d \gamma_{2}, \\
& =\int_{\gamma_{1}=0}^{2^{R_{c 1} H\left(b_{1} \mid b_{2}\right)}-1} \frac{1}{\Gamma_{1}} \exp \left(-\frac{\gamma_{1}}{\Gamma_{1}}\right) d \gamma_{1} \\
& =1-\exp \left[-\frac{2^{R_{c 1} H\left(b_{1} \mid b_{2}\right)}-1}{\Gamma_{1}}\right]
\end{aligned}
$$

and

$$
\begin{aligned}
& P_{2}=\int_{\gamma_{1}=2^{R_{c 1} H\left(b_{1} \mid b_{2}\right)}-1}^{2^{R_{c 1} H\left(b_{1}\right)}-1} \\
& \times \int_{\gamma_{2}=0}^{2{ }^{\left[R_{c 2} H\left(b_{1}, b_{2}\right)-\frac{R_{c 2}}{R_{c 1}} \log \left(1+\gamma_{1}\right)\right]}} p\left(\gamma_{1}\right) p\left(\gamma_{2}\right) d \gamma_{1} d \gamma_{2}, \\
& =\int_{\gamma_{1}=2^{R_{c 1} H\left(b_{1} \mid b_{2}\right)}-1}^{2^{R_{c 1} H\left(b_{1}\right)}-1} \\
& \times p\left(\gamma_{1}\right)\left[-\exp \left(-\frac{\gamma_{2}}{\Gamma_{2}}\right)\right]_{\gamma_{2}=0}^{\left[R_{c 2} H\left(b_{1}, b_{2}\right)-\frac{R_{c 2}}{\left.R_{c 1} \log \left(1+\gamma_{1}\right)\right]}-1\right.} d \gamma_{1}, \\
& =\int_{\gamma_{1}=2^{R_{c 1} H\left(b_{1} \mid b_{2}\right)_{-1}}}^{2^{R_{c 1} H\left(b_{1}\right)}-1} \frac{1}{\Gamma_{1}} \exp \left(-\frac{\gamma_{1}}{\Gamma_{1}}\right) \\
& \times\left[1-\exp \left(-\frac{2^{\left[R_{c 2} H\left(b_{1}, b_{2}\right)-\frac{R_{c 2}}{R_{c 1}} \log \left(1+\gamma_{1}\right)\right]}-1}{\Gamma_{2}}\right)\right] d \gamma_{1}, \\
& =\frac{1}{\Gamma_{1}} \int_{\gamma_{1}=2^{R_{c 1} H\left(b_{1} \mid b_{2}\right)}-1}^{2^{R_{c 1} H\left(b_{1}\right)}-1}\left[\exp \left(-\frac{\gamma_{1}}{\Gamma_{1}}\right)\right. \\
& \left.-\exp \left(-\frac{\gamma_{1}}{\Gamma_{1}}+\frac{1}{\Gamma_{2}}-\frac{2^{R_{c 2} H\left(b_{1}, b_{2}\right)}}{\Gamma_{2}\left(1+\gamma_{1}\right)^{\frac{R_{c 2}}{R_{c 1}}}}\right)\right] d \gamma_{1} .
\end{aligned}
$$

Unfortunately, the derivation of an explicit expression of (14) may not be possible. Hence, instead, the trapezoidal numerical integration [14] method is used to calculate $P_{2}$ in Section 4. 


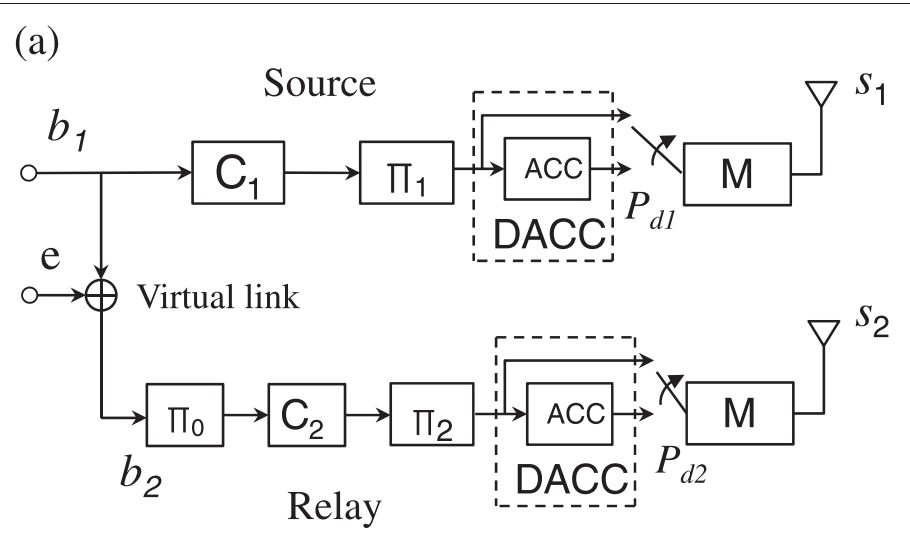

(b)

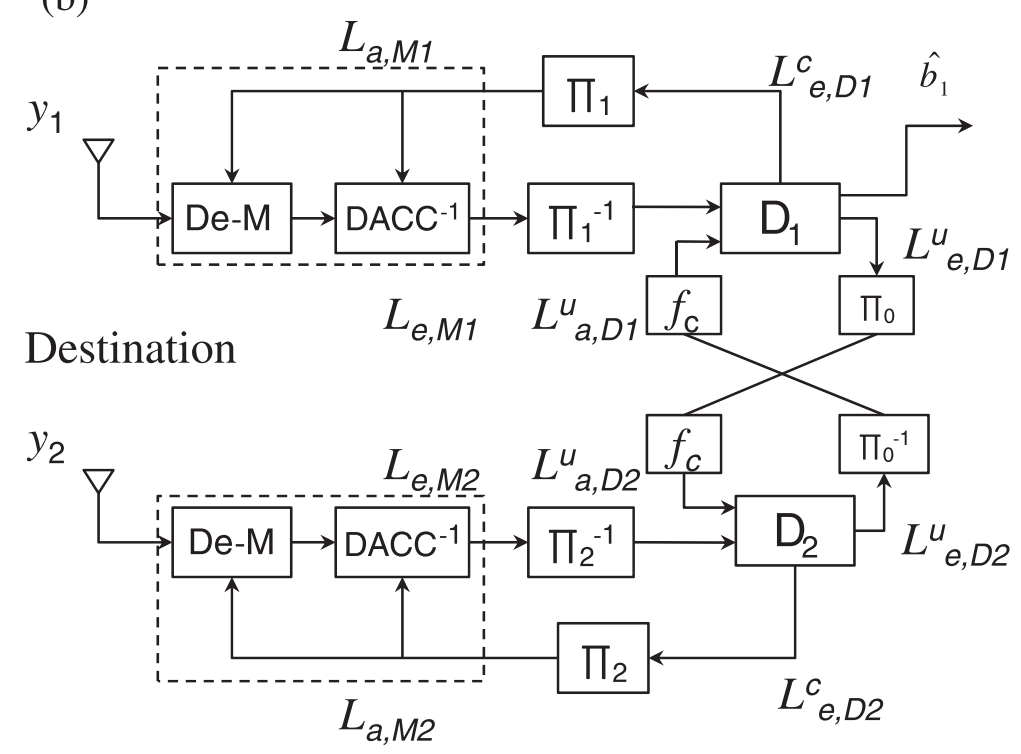

Figure 3 The schematic diagram of the proposed Slepian-Wolf relay system. (a) The source and the relay nodes. (b) The destination node. The BICM-ID demapper and the channel decoder exchange soft information via horizontal iterations (HI), while the two channel decoders also exchange information by exploiting the source-relay correlation, via vertical iterations (VI).

\subsubsection{Correlated links}

This section derives $P_{1}$ and $P_{2}$ taking into account the correlation $\rho=\left\langle h_{1} h_{2}^{*}\right\rangle$ of the fading variations. According to [13], when Link 1 and Link 2 are correlated, the signal amplitudes $A_{1}$ and $A_{2}\left(A_{i}=\sqrt{G_{i}\left|h_{i}\right|^{2} E_{s, i}}\right)$ follow the joint pdf $p\left(A_{1}, A_{2}\right)$, as shown in (15):

$$
\begin{aligned}
p\left(A_{1}, A_{2}\right)= & \frac{4 A_{1} A_{2}}{P_{r 1} P_{r 2}\left(1-|\rho|^{2}\right)} I_{0}\left[\frac{2|\rho| A_{1} A_{2}}{\sqrt{P_{r 1} P_{r 2}}\left(1-|\rho|^{2}\right)}\right] \\
& \times \exp \left[-\frac{1}{1-|\rho|^{2}}\left(\frac{A_{1}^{2}}{P_{r 1}}+\frac{A_{2}^{2}}{P_{r 2}}\right)\right],
\end{aligned}
$$

where $I_{0}(\cdot)$ is the zeroth order modified Bessel's function of the first kind. We define the average SNR of the $i$ th channel as $\Gamma_{i}=P_{r i} /\left(2 \sigma^{2}\right)$, where $P_{r i}=\left\langle\left|h_{i}\right|^{2} E_{i}\right\rangle$, denoting the average received signal power of Link i. Since $I_{0}(x)$ can be expanded into a series $I_{0}(x)=\sum_{n=0}^{\infty} \frac{(x / 2)^{2 n}}{(n !)^{2}}$, (15) can be re-written as [15]

$$
\begin{aligned}
p\left(A_{1}, A_{2}\right)= & \frac{4 A_{1} A_{2}}{P_{r 1} P_{r 2}\left(1-|\rho|^{2}\right)} \exp \left(-\frac{A_{1}^{2} / P_{r 1}}{1-|\rho|^{2}}-\frac{A_{2}^{2} / P_{r 2}}{1-|\rho|^{2}}\right) \\
& \sum_{n=0}^{\infty} \frac{1}{(n !)^{2}}\left(\frac{|\rho| A_{1} A_{2}}{\sqrt{P_{r 1} P_{r 2}}\left(1-|\rho|^{2}\right)}\right)^{2 n}, \\
= & \sum_{n=0}^{\infty} q_{1}^{(n)} q_{2}^{(n)}
\end{aligned}
$$

where $q_{1}^{(n)}$ and $q_{2}^{(n)}$ can be expressed as

$$
q_{1}^{(n)}=\frac{2 A_{1}^{2 n+1}|\rho|^{n}}{P_{r 1}^{n+1}\left(1-|\rho|^{2}\right)^{n+1 / 2}} \exp \left(-\frac{A_{1}^{2} / P_{r 1}}{1-|\rho|^{2}}\right)\left(\frac{1}{n !}\right)
$$




$$
q_{2}^{(n)}=\frac{2 A_{2}^{2 n+1}|\rho|^{n}}{P_{r 2}^{n+1}\left(1-|\rho|^{2}\right)^{n+1 / 2}} \exp \left(-\frac{A_{2}^{2} / P_{r 2}}{1-|\rho|^{2}}\right)\left(\frac{1}{n !}\right) .
$$

Since $p\left(A_{1}, A_{2}\right)$ can be factored into a product of two independent terms, each for its corresponding random variable, it is easy to calculate $P_{1}$ and $P_{2}$ by substituting (16)-(18) and $\gamma_{i}=A_{i}^{2} /\left(2 \sigma^{2}\right)$ into (13) and (14), with the aid of the trapezoidal methods.

\subsubsection{Outage of MRC}

For a comparison with our proposed Slepian-Wolf relay system, the outage probability of the maximum ratio combining (MRC) scheme is derived with the assumptions that $p_{e}=0^{\mathrm{b}}, \rho=0$ and $\Gamma_{1}=\Gamma_{2}$. It is well known that the output of the MRC combiner is a weighted sum of signals received via all the transmission links. The $p d f p_{\gamma_{\Sigma}}(\gamma)$ of the instantaneous SNR $\gamma$ in the block Rayleigh channel after the MRC combining is given by [16]

$$
p_{\gamma_{\Sigma}}(\gamma)=\frac{\gamma^{N-1} \exp \left(-\frac{\gamma}{\Gamma}\right)}{\Gamma^{N}(N-1) !}
$$

where $N$ denotes the diversity order, and we have assumed each channel has the same average SNR $\Gamma$. The outage probability of MRC is defined as the probability that the instantaneous SNR after combining is less than a given threshold. For a fair comparison, the threshold of the transmission rate is chosen to be $R_{c 1} H\left(b_{1}\right)$. Then, the outage probability of the 2 nd order MRC diversity can be calculated as follows

$$
\begin{aligned}
P_{\text {out }, \mathrm{mrc}}= & \int_{\gamma=0}^{2^{R_{c 1} H\left(b_{1}\right)}-1} p_{\gamma_{\Sigma}}(\gamma) d \gamma_{1}, \\
= & \int_{\gamma=0}^{2_{c 1}^{R_{c 1} H\left(b_{1}\right)}-1} \frac{\gamma \exp \left(-\frac{\gamma}{\Gamma}\right)}{\Gamma^{2}} d \gamma, \\
= & 1-\exp \left(\frac{1-2^{R_{c 1} H\left(b_{1}\right)}}{\Gamma}\right) \\
& \times \sum_{k=1}^{2} \frac{\left[\left(2^{R_{c 1} H\left(b_{1}\right)}-1\right) / \Gamma\right]^{k-1}}{(k-1) !} .
\end{aligned}
$$

\section{Asymptotic tendency analysis}

\subsection{Tendency $1: \ln$ the case $p_{e}=0$}

This section provides the proof of the fact that, when $b_{1}$ and $b_{2}$ are fully correlated $\left(p_{e}=0\right)$, the 2nd order diversity of the outage curves can be achieved. In this case, we have $H\left(b_{1} \mid b_{2}\right)=0, H\left(b_{1}, b_{2}\right)=1$ and the value of $P_{1}$ is always equal to 0 as found from (13). Hence, the outage probability is determined by $P_{2}$ only. By assuming that the fading variations of the two links are statistically independent $(\rho=0)$ and $R_{c 1}=R_{c 2}=1$, the derivation of $P_{2}$ can be reduced to

$$
\begin{aligned}
P_{2}= & \int_{\gamma_{1}=0}^{1} \int_{\gamma_{2}=0}^{2^{\left[1-\log _{2}\left(1+\gamma_{1}\right)\right]}-1} p\left(\gamma_{1}\right) p\left(\gamma_{2}\right) d \gamma_{1} d \gamma_{2}, \\
= & \int_{0}^{1} p\left(\gamma_{1}\right) d \gamma_{1}\left[-\exp \left(-\frac{\gamma_{2}}{\Gamma_{2}}\right)\right]_{0}^{2^{\left[1-\log _{2}\left(1+\gamma_{1}\right)\right]}-1} \\
= & \frac{1}{\Gamma_{1}} \int_{0}^{1}\left[\exp \left(-\frac{\gamma_{1}}{\Gamma_{1}}\right)\right. \\
& \left.-\exp \left(-\frac{\gamma_{1}}{\Gamma_{1}}+\frac{1}{\Gamma_{2}}-\frac{2}{\Gamma_{2}\left(1+\gamma_{1}\right)}\right)\right] d \gamma_{1} .
\end{aligned}
$$

By using (22) when $x$ is very small:

$$
\mathrm{e}^{-x}=\sum_{n=0}^{\infty} \frac{(-x)^{n}}{n !} \approx 1-x
$$

(21) can be approximated as

$$
\begin{aligned}
P_{2} \approx & \frac{1}{\Gamma_{1}} \int_{0}^{1}\left[\left(1-\frac{\gamma_{1}}{\Gamma_{1}}\right)\right. \\
& \left.-\left(1-\frac{\gamma_{1}}{\Gamma_{1}}+\frac{1}{\Gamma_{2}}-\frac{2}{\Gamma_{2}\left(1+\gamma_{1}\right)}\right)\right] d \gamma_{1} \\
= & \frac{1}{\Gamma_{1}} \int_{0}^{1}\left[-\frac{1}{\Gamma_{2}}+\frac{2}{\Gamma_{2}\left(1+\gamma_{1}\right)}\right] d \gamma_{1} \\
= & \frac{1}{\Gamma_{1}}\left[\frac{2 \ln \left(1+\gamma_{1}\right)-\gamma_{1}}{\Gamma_{2}}\right]_{0}^{1} \\
= & \frac{2 \ln 2-1}{\Gamma_{1} \Gamma_{2}} .
\end{aligned}
$$

Obviously, the final result shows that with $p_{e}=0$, the outage probability curve is inversely proportional to the product of $\Gamma_{1}$ and $\Gamma_{2}$, which achieves the 2nd order diversity.

\subsection{Tendency 2: Slepian-Wolf relay versus MRC}

In this section, the proof of the advantage of the SlepianWolf relay system over MRC is presented, assuming that $\Gamma_{1}=\Gamma_{2}$ in both the schemes with $p_{e}=0, \rho=0$ and $R_{c 1}=R_{c 2}=1$. Then, (14) and (20) can be further reduced to

$$
\begin{aligned}
P_{2}=\frac{1}{\Gamma_{1}} & \int_{\gamma_{1}=0}^{1}\left\{\exp \left(-\frac{\gamma_{1}}{\Gamma_{1}}\right)\right. \\
& \left.\times-\exp \left[\frac{1}{\Gamma_{1}}\left(1-\gamma_{1}-\frac{2}{1+\gamma_{1}}\right)\right]\right\} d \gamma_{1},
\end{aligned}
$$

and

$$
P_{\text {out }, \mathrm{mrc}}=\frac{1}{\Gamma_{1}} \int_{\gamma_{1}=0}^{1} \frac{\gamma_{1}}{\Gamma_{1}} \exp \left(-\frac{\gamma_{1}}{\Gamma_{1}}\right) d \gamma_{1}
$$


To prove that $P_{\text {out,mrc }}-P_{2}>0$, we define $P_{\text {gap }}=P_{\text {out,mrc }}-$ $P_{2}$

$$
\begin{aligned}
P_{\text {gap }}= & \frac{1}{\Gamma_{1}} \int_{\gamma_{1}=0}^{1}\left\{\exp \left[\frac{1}{\Gamma_{1}}\left(1-\gamma_{1}-\frac{2}{1+\gamma_{1}}\right)\right]\right. \\
& \left.+\left(\frac{\gamma_{1}}{\Gamma_{1}}-1\right) \exp \left(-\frac{\gamma_{1}}{\Gamma_{1}}\right)\right\} d \gamma_{1} \\
= & \frac{1}{\Gamma_{1}}\left\{\int_{\gamma_{1}=0}^{1} \exp \left[\frac{1}{\Gamma_{1}}\left(1-\gamma_{1}-\frac{2}{1+\gamma_{1}}\right)\right]\right. \\
& \left.\times d \gamma_{1}-\exp \left(-\frac{1}{\Gamma_{1}}\right)\right\} .
\end{aligned}
$$

Let $y_{1}(x)=\exp \left(1-x-\frac{2}{1+x}\right)$. It is found that $y_{1}(x) \geqslant$ -1 within the range of $[0,1]$ if $y_{1}(x)$ is concave, since $y_{1}(x) \geqslant \min \left\{y_{1}(0), y_{1}(1)\right\}=-1$, according to the property of the concave function. $y_{1}(x)$ can be proven to be concave by showing that

$$
\begin{aligned}
y_{1}(x)^{\prime \prime}= & \exp \left(1-x-\frac{2}{1+x}\right)\left[\frac{2}{(1+x)^{2}}-1\right]^{2} \\
& -\frac{4 \exp \left(1-x-\frac{2}{1+x}\right)}{(1+x)^{3}}<0 .
\end{aligned}
$$

By ignoring the common exponential terms in (27), because they are positive, it is found that giving a proof to (27) is equivalent to proving that $y_{2}(x)=$ $\left[2-(1+x)^{2}\right]^{2}-4(1+x)<0$. Let $t=1+x(t \in[1,2])$. Then, $y_{2}(t)=\left(2-t^{2}\right)^{2}-4 t=t^{4}-4 t^{2}-4 t+4$. The second-order derivative of $y_{2}(t)$ can be expressed as

$$
y_{2}(t)^{\prime \prime}=12 t^{2}-8
$$

Obviously, $y_{2}(t)^{\prime \prime}>0$ within the range of $[1,2]$. Therefore $y_{2}(t)$ is convex, and $y_{2}(t)<\max \left\{y_{2}(1), y_{2}(2)\right\}=-3$. Hence, $y_{2}(t)<0$, which is equivalent to $y_{1}(x)^{\prime \prime}<0$. Now $y_{1}(x)$ is proved to be concave, and consequently $P_{\text {gap }}$ is proved to be positive. As a result, Slepian-Wolf relaying yields a lower outage.

\subsection{Tendency $3:$ in the case $p_{e} \neq 0$}

When $b_{1}$ and $b_{2}$ are not fully correlated $\left(p_{e} \neq 0\right)$, the asymptotic tendency of the outage curve is proven to converge into that of the 1 st order diversity. With $\rho=0$, when $\Gamma_{1} \rightarrow \infty$ and $\Gamma_{2} \rightarrow \infty, P_{2} \rightarrow 0$ according to (11). Therefore only $P_{1}$ dominates the outage probability. Assuming $R_{c 1}=1$, (10) can be approximated by using (22), as

$$
\begin{aligned}
P_{1} & =1-\exp \left[-\frac{2^{H\left(b_{1} \mid b_{2}\right)}-1}{\Gamma_{1}}\right] \\
& \approx \frac{2^{H\left(b_{1} \mid b_{2}\right)}-1}{\Gamma_{1}} .
\end{aligned}
$$

Obviously, when the average SNRs $\Gamma_{1}$ and $\Gamma_{2}$ become large, the value of $P_{1}$ is inversely in proportion to $\Gamma_{1}$ and hence the diversity order converges into 1 .

\subsection{Tendency 4: correlated link variation}

In the presence of the correlation $\rho \neq 0$ of the fading variation, regardless of the source correlation $\mu$, increasing the average SNRs $\Gamma_{1}$ and $\Gamma_{2}$, or equivalently increasing $P_{r 1}$ and $P_{r 2}$ yields [15]:

$$
\frac{2|\rho| A_{1} A_{2}}{\sqrt{P_{r 1} P_{r 2}}\left(1-|\rho|^{2}\right)} \approx 0 \quad\left(P_{r 1} \rightarrow \infty, P_{r 2} \rightarrow \infty\right)
$$

Hence, with $I_{0}(0) \rightarrow 1$, (15) can be approximated as

$$
\begin{aligned}
p\left(A_{1}, A_{2}\right) \approx & \frac{4 A_{1} A_{2}}{P_{r 1} P_{r 2}\left(1-|\rho|^{2}\right)} \exp \left(-\frac{A_{1}^{2} / P_{r 1}}{1-|\rho|^{2}}-\frac{A_{2}^{2} / P_{r 2}}{1-|\rho|^{2}}\right) \\
= & \frac{2 A_{1}}{P_{r 1} \sqrt{1-|\rho|^{2}}} \exp \left(-\frac{A_{1}^{2}}{P_{r 1}\left(1-|\rho|^{2}\right)}\right) \\
& \frac{2 A_{2}}{P_{r 2} \sqrt{1-|\rho|^{2}}} \exp \left(-\frac{A_{2}^{2}}{P_{r 2}\left(1-|\rho|^{2}\right)}\right) \\
= & p\left(A_{1}^{\prime}\right) p\left(A_{2}^{\prime}\right),
\end{aligned}
$$

where $A_{1}^{\prime}=A_{1} \sqrt{1-|\rho|^{2}}$ and $A_{2}^{\prime}=A_{2} \sqrt{1-|\rho|^{2}}$, with $P_{r 1}^{\prime}=P_{r 1}\left(1-|\rho|^{2}\right)$ and $P_{r 2}^{\prime}=P_{r 2}\left(1-|\rho|^{2}\right)$. Obviously, $p\left(A_{i}^{\prime}\right)=\frac{2 A_{i}^{\prime}}{P_{r i}^{\prime}} \exp \left(-\frac{A_{i}^{\prime 2}}{P_{r i}^{\prime}}\right)$, which corresponds to the well-known $p d f$ of the Rayleigh-distributed signal envelope of Link $i$ [16]. Hence, with $P_{r 1} \rightarrow \infty$ and $P_{r 2} \rightarrow \infty$ (equivalently, $P_{r 1}^{\prime} \rightarrow \infty$ and $P_{r 2}^{\prime} \rightarrow \infty$ ), the asymptotic property of the outage probability exhibits the same tendency as in the case of independent channels, which indicates that the tendency of the diversity order only depends on the source correlation.

\section{BICM-ID based Slepian-Wolf relay system}

In this section, we apply the Slepian-Wolf relay technique presented in [6] to a system utilizing BICM-ID for Link 1 and Link 2 transmission [17], to demonstrate how the correlation knowledge can be utilized in [6]. The FER performance is then compared with the theoretical results in the next section. The block diagrams of the BICM-ID based Slepian-Wolf relay system are shown in Figure 3a,b. The system block diagram of the source and relay nodes is shown in Figure 3a, where the original information bits $b_{1}$ at the source node are channel-encoded by $C_{1}$, interleaved by $\Pi_{1}$, and further encoded by a doped accumulator (DACC). DACC has the same structure as memory-1 half rate systematic recursive convolutional code (SRCC), however, its outputs are mostly the systematic bits where only the every $P_{d 1}$ th systematic bit is replaced by the accumulated coded bit ( $P_{d 1}$ denotes the doping ratio). It should be noticed that DACC does not change the code rate. It should be noticed further that the extrinsic information transfer (EXIT) curve of the corresponding decoder $\mathrm{DACC}^{-1}$ reaches until a point very close to the $(1,1)$ mutual information point $[8,18]$. Finally, the bit stream 
output from the DACC is mapped onto symbols $s_{1}$ according to the specified modulation rule and broadcasted to both the relay and destination nodes during the first time slot.

After receiving the signals via the intra-link, the relay node aims to recover the original information bits, according to the DF strategy. However, the relay is not necessarily able to totally eliminate the errors happening in the intra-link [6], and hence some errors may remain in the recovered information $b_{2}$. The purpose of the simulation is to verify the consistency in the performance tendency to the theoretical results, and hence the bit-flipping model is used to simplify the intra-link transmission errors occurring in the information part of the recovered bit stream, as shown in Figure $3 \mathrm{a}^{\mathrm{c}}$. The recovered information bit stream has to be interleaved by $\Pi_{0}$ before being re-encoded by $C_{2}$. This process plays the crucial role in the sense that it converts the relay system to a form of distributed turbo code (DTC) [19]. The re-encoded stream is further interleaved by $\Pi_{2}$, and fed into DACC (with a doping ratio $P_{d 2}$ ). Finally, the DACC output is modulated with a specified mapping rule and the symbols $s_{2}$ at the relay node are sent to the destination during the second time slot.

At the destination as shown in Figure 3b, the received signals from both Link 1 and Link 2 are first demapped based on the BICM-ID principle, as [20,21]:

$$
\begin{aligned}
L_{e}\left(b_{v}\right) & =\ln \frac{P\left(b_{v}=1 \mid y\right)}{P\left(b_{v}=0 \mid y\right)} \\
& =\ln \frac{\sum_{s \in S_{1}}\left\{\exp \left(-\frac{|y-\sqrt{G} h|^{2}}{2 \sigma^{2}}\right) \prod_{w \neq v}^{M} \exp \left[b_{w} L_{a}\left(b_{w}\right)\right]\right\}}{\sum_{s \in S_{0}}\left\{\exp \left(-\frac{|y-\sqrt{G} h|^{2}}{2 \sigma^{2}}\right) \prod_{w \neq \nu}^{M} \exp \left[b_{w} L_{a}\left(b_{w}\right)\right]\right\}},
\end{aligned}
$$

where $v$ is the position of the detecting bit in the symbold ${ }^{\mathrm{d}}$. $M$ is the number of bits per symbol and $L_{a}\left(b_{w}\right)$ denotes the a priori LLRs of the $w$ th bit in symbol $s$, fed back from the channel decoder. DACC ${ }^{-1}$, and the decoders $D_{1}$ and $D_{2}$ for the channel encoders $C_{1}$ and $C_{2}$, respectively, use the Bahl, Cocke, Jelinek, and Raviv (BCJR) algorithm [22]. $S_{0}$ and $S_{1}$ are the sets of symbols having the $v$ th bit being zero or one, respectively. The output extrinsic LLRs of the demapper are brought to DACC ${ }^{-1}$, followed by the channel decoder via $\Pi_{1}$ and $\Pi_{2}$ for the first and second time slots, respectively. This process is referred to as the horizontal iterations (HI), as shown in Figure $3 \mathrm{~b}$.

Furthermore, the channel decoders $D_{1}$ and $D_{2}$ also exchange soft information with each other. This process is referred to as the vertical iterations (VI). It should be noticed that the LLR updating function is used in the $\mathrm{VI}$, as illustrated in Figure $3 \mathrm{~b}$ ), where the knowledge of correlation $\mu$ (equivalently, the information bit flipping probability $p_{e}$ of the intra-link) is utilized. Furthermore according to [6], the intra-link error probability representing the correlation can be estimated at the destination, as

$$
\hat{p}_{e}=\frac{1}{N} \sum_{n=1}^{N} \frac{e^{L_{p, D_{1}}^{u}}+e^{L_{p, D_{2}}^{u}}}{\left(1+e^{L_{p, D_{1}}^{u}}\right)\left(1+e^{L_{p, D_{2}}^{u}}\right)},
$$

where $\hat{p}_{e}$ is the estimate of $p_{e}$, and $L_{p, D 1}^{u}$ and $L_{p, D 2}^{u}$ are the a posteriori LLRs of the uncoded information bits, output from the decoders $D_{1}$ and $D_{2}$, respectively. A threshold has to be set appropriately, as shown in [6], to select only the reliable LLRs when calculating $\hat{p}_{e}$ using (33). $N$ represents the number of the reliable a posteriori LLR pairs $L_{p, D 1}^{u}$ and $L_{p, D 2}^{u}$. The estimate $\hat{p}_{e}$ of the intra-link error probability $p_{e}$ is used to modify the bit probability, taking into account the intra-link errors, as [8]:

$$
\begin{aligned}
& \operatorname{Pr}\left(b_{2}=0\right)=\left(1-p_{e}\right) \operatorname{Pr}\left(b_{1}=0\right)+p_{e} \operatorname{Pr}\left(b_{1}=1\right), \\
& \operatorname{Pr}\left(b_{2}=1\right)=\left(1-p_{e}\right) \operatorname{Pr}\left(b_{1}=1\right)+p_{e} \operatorname{Pr}\left(b_{1}=0\right),
\end{aligned}
$$

which leads to the LLR updating function $f_{c}$ expressed as follows [23]:

$$
f_{c}(x)=\ln \frac{\left(1-\hat{p}_{e}\right) \cdot \exp (x)+\hat{p}_{e}}{\left(1-\hat{p}_{e}\right)+\hat{p}_{e} \cdot \exp (x)} .
$$

In (35), $x$ denotes the extrinsic LLRs of the information bits, obtained by $D_{1}$ and $D_{2}$, and the output of $f_{c}$ are the updated LLRs by exploiting $\hat{p}_{e}$ as the correlation knowledge of the intra-link. The VI operations at the receiver can be expressed as:

$$
\begin{aligned}
& L_{a, D_{1}}^{u}=f_{c}\left\{\Pi_{0}^{-1}\left(L_{e, D_{2}}^{u}\right)\right\}, \\
& L_{a, D_{2}}^{u}=f_{c}\left\{\Pi_{0}\left(L_{e, D_{1}}^{u}\right)\right\} .
\end{aligned}
$$

\section{Numerical results}

In this section, the numerical results of the theoretical outage probability calculation and the FER performance of the BICM-ID based Slepian-Wolf relay system obtained through simulations are presented. In the simulations for the BICM-ID based Slepian-Wolf relay system, we use the half rate non-recursive systematic convolutional code with generator polynomials of $(3,2)_{8}$ for both $C_{1}$ and $C_{2}$. Non-Gray quaternary-phase-shift-keying (QPSK) was used for both the Link 1 and Link 2 transmission ${ }^{\mathrm{e}}$. Hence, the coefficients $R_{c i}(i=1,2)$ representing channel coding rate and modulation multiplicity is equal to one $\left(=\frac{1}{2} \times 2\right)$ in the theoretical calculations of the outage probability. The interleaver length is 4000 and the doping ratio $P_{d 1}$ and $P_{d 2}$ are set to five in the simulations. The LLR threshold for (33) was set to one, as in [6], to select the reliable LLRs output from $D_{1}$ and $D_{2}$.

Figure 4 shows the theoretical outage probabilities of the proposed Slepian-Wolf relay system, in the case $\rho=0$ 


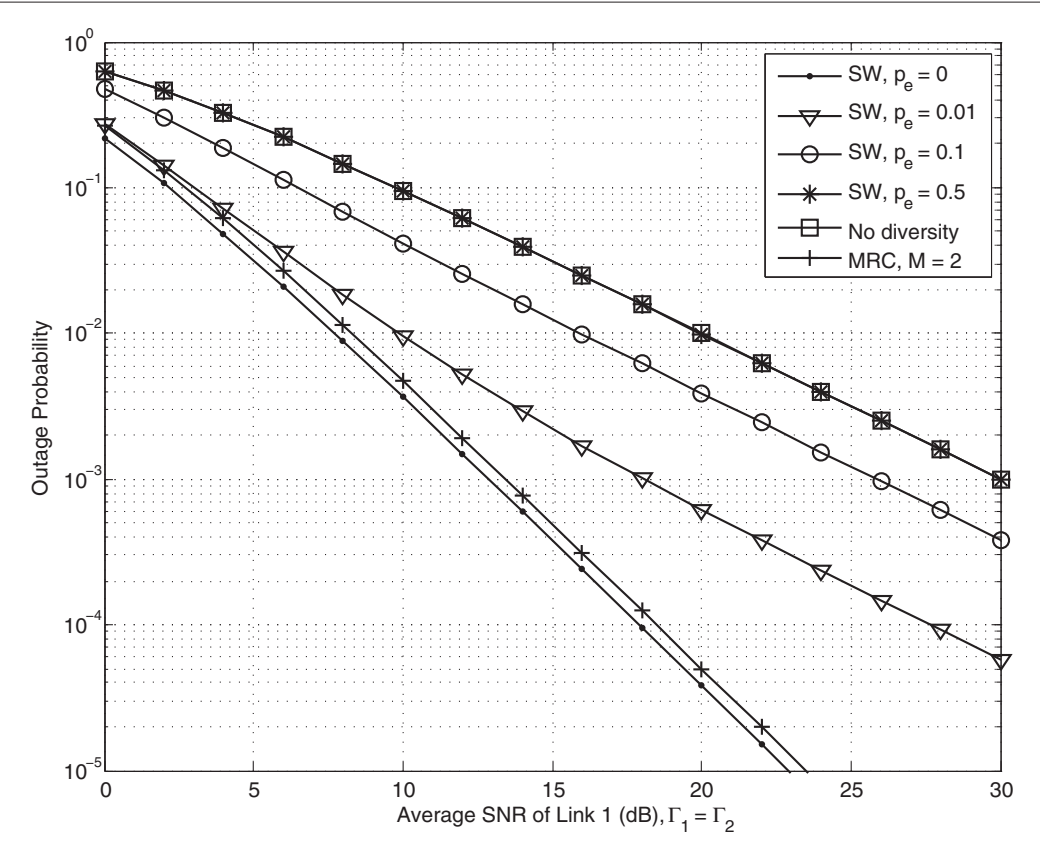

Figure 4 The theoretical outage probability of the proposed relay transmission model, $\Gamma_{1}=\Gamma_{2}$, and $\rho=0$, with $p_{e}$ as a parameter. The 2nd order diversity can be achieved only if $b_{1}$ and $b_{2}$ are fully correlated $\left(p_{e}=0\right)$ with $\rho=0$.

and average SNRs $\Gamma_{1}=\Gamma_{2}$. It is found that, only when $b_{1}$ and $b_{2}$ are fully correlated $\left(p_{e}=0\right)$, the 2 nd order diversity can be achieved, which is consistent to the asymptotic tendency analysis presented in Section 4.1. Moreover, it should be noticed that the Slepian-Wolf relay system can achieve slightly better outage performance than that with MRC, which is also consistent to the mathematical proof presented in Section 4.2. However, in the case when $p_{e} \neq$ 0 , the diversity order of the outage curves always plateaus at one as the average SNR increases. This asymptotic tendency agrees with the mathematical proof presented in Section 4.3. When $b_{1}$ and $b_{2}$ are completely independent $\left(p_{e}=0.5\right)$, obviously, the outage curves of the Slepian-Wolf relay is the same as that without diversity.

Figure 5 demonstrates the FER performance of the BICM-ID based Slepian-Wolf relay system $(\rho=0)$, where the theoretical outage curve is also plotted for comparison. It is found that the FER and the theoretical outage curves exhibit the same decay, however, there is a $2-3 \mathrm{~dB}$ gap in average SNR between them. This is because the BICM-ID technique used in this example does not achieve close-capacity performance. This indicates that there is a possibility that the gap can further be reduced by using very close-capacity achieving techniques $[21,24]$.

Figure 6 shows the impact of the relay location on the outage probability as a function of the distance ratio $d_{2} / d_{1}$, by assuming that the relay location is in a line between source and destination nodes. According to (1), $\Gamma_{2}=\Gamma_{1}+10 \log _{10}\left[\left(d_{1} / d_{2}\right)^{3.52}\right](\mathrm{dB})$. Because of the very stable intra-link assumption that we made in the theoretical analysis, in the simulation for the impact evaluation of the relay location, we assume an AWGN source-relay link ${ }^{\mathrm{f}}$. The received average SNR at the relay is denoted by $\Gamma_{R}$, and $\Gamma_{R}=\Gamma_{1}+10 \log _{10}\left[\left(d_{1} /\left(d_{1}-d_{2}\right)\right)^{3.52}\right](\mathrm{dB})$. Furthermore, we assume the Slepian-Wolf relaying technique presented in [25], where the coding and modulation parameters are exactly the same as that described in the previous section. The only difference from the technique presented in the previous section is that in [25], the intra-link signal detection process is also included, where instead of performing fully iterative decoding at the relay, only systematic part is extracted (no decoding for $C_{1}$ is performed after viterbi decoding of DACC). This significantly reduces the computational complexity of the relay [25].

We first obtained the bit error rate (BER) curve of the intra-link transmission via simulations ${ }^{g}$. The SNR values were calculated at different locations using the equation shown above, and then converted into $p_{e}$ referring to the simulation result. With the fixed $p_{e}$ value, obtained via the methodology described above, we evaluated the FER performance via simulations, where Link 1 and Link 2 were assumed to suffer from statistically independent block Rayleigh fading. The FER curves are shown in Figure 6 for $\Gamma_{1}=3 \mathrm{~dB}$, together with the theoretical outage curve.

Interestingly, it is found that there is an optimal relay location. If the relay location is too close to the source, the FER performance is worse than that at the optimal 


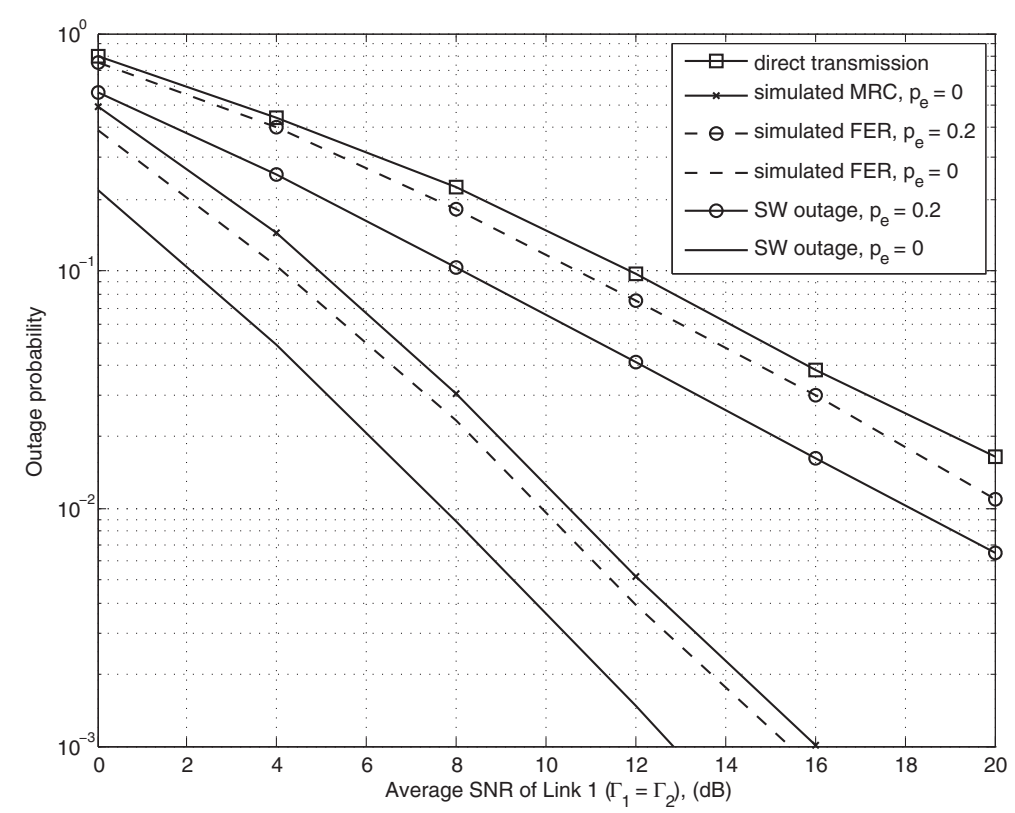

Figure 5 Comparison of the theoretical outage probability and the FER of the BICM-ID based Slepian-Wolf relay system. The performance tendencies of the theoretical outage probability and the FER of the BICM-ID based relay system are consistent with each other.

location. This tendency is consistent to the theoretical outage curve also shown in Figure 6. In fact, there are two factors that improve the FER/outage performance: one is the correlation exploitation, and the other is the energy of each link. Let the distance between the source and the optimal location be denoted by $d_{\mathrm{opt}}$. At $d_{\mathrm{opt}}$, the combined effect is maximized, while the contribution of Link 2 energy tends to be smaller when $d_{1}-d_{2}<d_{\mathrm{opt}}$ (In this case, the $p_{e}$ value is already very small and hence the contribution of correlation is fully exploited). It is also found that there is a gap between the FER simulation result and theoretical outage curve, because the

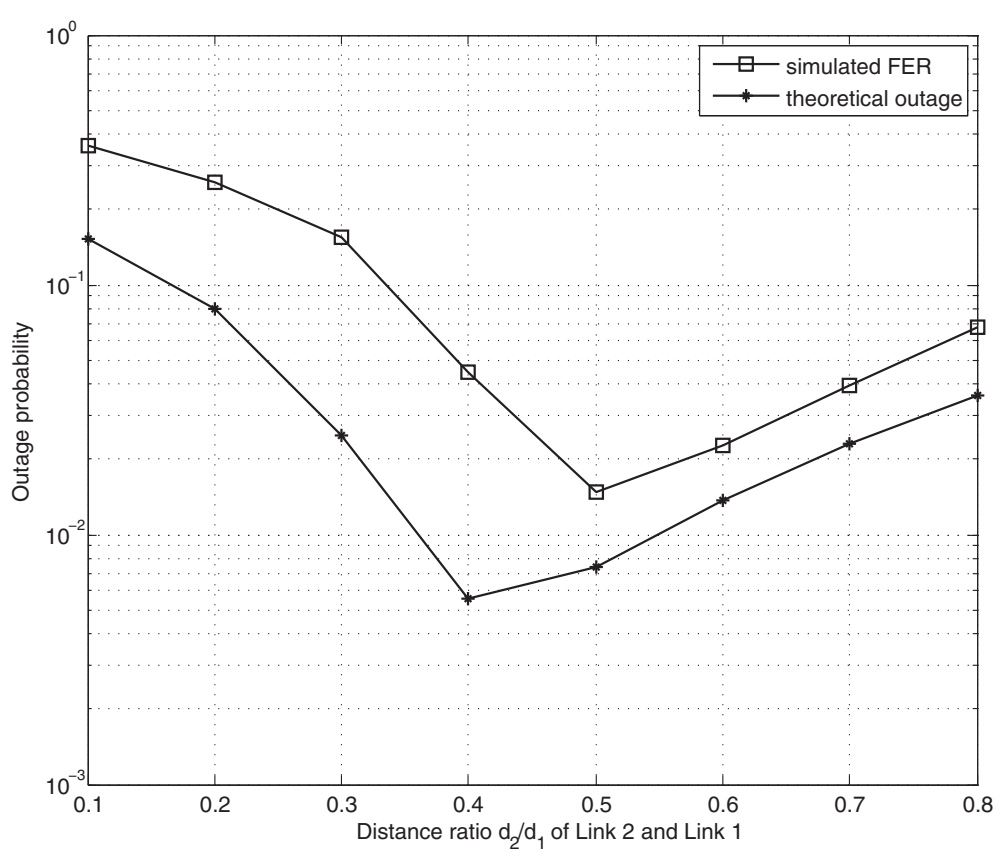

Figure 6 Outage probability versus the distance ratio. The $p_{e}$ value follows the distribution of the BER of the intra-link.. 


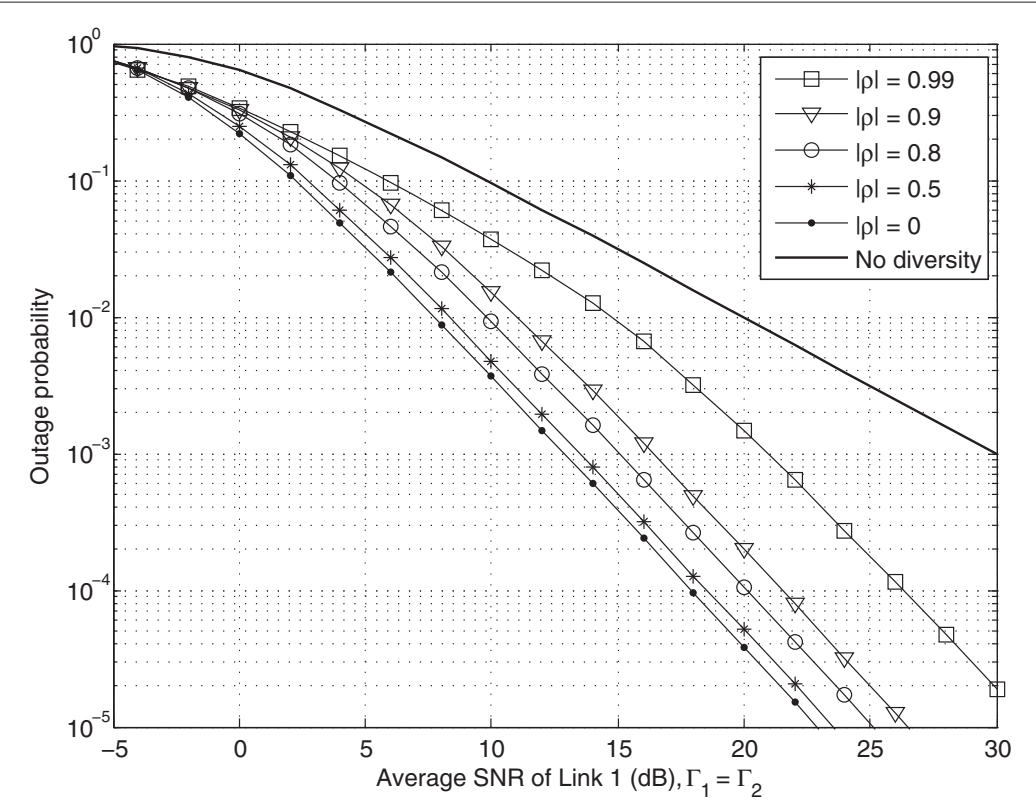

Figure $\mathbf{7}$ The theoretical outage probability when fading of the two links is correlated and $\boldsymbol{p}_{\boldsymbol{e}} \mathbf{=} \mathbf{0}$. When $b_{1}$ and $b_{2}$ are fully correlated, the 2nd order diversity can still be achieved if $|\rho|<1$.

BICM-ID relay in this article does not achieve closecapacity performance as stated before.

Figure 7 shows the theoretical outage curves in the presence of fading correlation $\rho$ between Link 1 and Link 2 while the source bits $b_{1}$ and $b_{2}$ are fully correlated $\left(p_{e}=\right.$ $0)$. Obviously, the larger the correlation of the fading variation, the larger the outage probability. This is consistent to the theoretical result provided in Section 4.4 that the 2nd order diversity can finally be achieved with arbitrary value of $|\rho| \neq 1$, by increasing the average SNRs.

\section{Conclusions}

In this study, we have theoretically analyzed the outage probability and its asymptotic properties of a simple oneway relay transmission system allowing intra-link errors, where the source-relay correlation can be exploited in the joint decoding process at the destination, based on the Slepian-Wolf theorem. The outage probability can be calculated by a set of double integrals of the admissible rate region with respect to the $p d f$ of the instantaneous SNRs of the transmission links, identified by the Slepian-Wolf theorem. It has been found through the asymptotic tendency analysis that the 2nd order diversity can be achieved only when the information bit streams at the source and the relay are fully correlated. Otherwise, it converges into one as the average SNRs of the source-destination and/or relay-destination links increase. Interestingly, the Slepian-Wolf relay system's outage probability is found to be smaller than the case where the signals received via the two links are maximum-ratio-combined before decoding.
We have provided a mathematical proof of this discovery. However, this proof has been given to only the case where the two links suffer from independent block Rayleigh fading with the same average SNR. Hence, whether or not this discovery commonly holds for the arbitrary distance ratio $d_{2} / d_{1}$, and/or for correlated fading variation, is still an open hypothesis. The correlation $\rho$ of the fading variation [15] of the source-destination and relay-destination links were also taken into account, in addition to the sourcerelay information correlation $\mu=\left\langle\left(2 b_{1}-1\right)\left(2 b_{2}-1\right)\right\rangle$. Interestingly, it has been found that the diversity order is only determined by the information correlation and is independent of the link variation correlation, so far as $|\rho|<1$. It has to be noted that the intra-link transmission is parameterized by a bit-flipping probability $p_{e}$ in this article. This is because $p_{e}$ is the bit error probability of the information part after decoding at the relay, which depends on a lot of parameters related to the intralink transmission. If the probability density function $(p d f)$ of $p_{e}$ is known, obviously it is possible to calculate the outage probability in the case that the three links are all time-varying. However, this is left as a future study.

This article also compared the FER of a BICM-ID based Slepian-Wolf relay system, as an example of our proposed technique, with the theoretical outage probability for $\rho=$ 0 . The decay of the FER and outage curves are consistent with each other, however, with a $2-3 \mathrm{~dB}$ gap in average SNR between them. This is because the BICM-ID technique used in this example does not achieve close-capacity performance, and there is a probability that the gap 
can be reduced by utilizing very close-capacity achieving code.

\section{Endnotes}

${ }^{\mathrm{a}} \mathrm{A}$ Gaussian codebook is assumed for channel coding. ${ }^{b}$ Even without the interleaver $\Pi_{0}$ at the relay node, a serious error propagation is expected in the case of MRC, if $p_{e} \neq 0$, due to the use of DACC. Hence, performing MRC at the destination by ignoring the intra-link errors even degrades the performance. However, without DACC, HI cannot reach a point in the extrinsic information transfer (EXIT) chart close enough to the $(1,1)$ mutual information point. The terminologies "DACC" and "HI" are introduced in Section 5.

${ }^{\mathrm{c}}$ This indicates that in the simulations, we did not perform actual decoding and demapping processes at the relay.

${ }^{\mathrm{d}}$ Equation (32) holds both for Link 1 and Link 2. Therefore, for the sake of simplicity, we omitted the link index from the variables.

${ }^{\mathrm{e}}$ Through the EXIT chart analysis conducted as a preliminary study, the demapper and the decoder's EXIT curves are found to be well matched with the transmission chain parameters described above, even though the codes used are very simple and easy to decode.

${ }^{\mathrm{f}}$ The $p_{e}$ value is a parameter which follows the distribution of the bit error rate of the intra-link.

${ }^{\mathrm{g}}$ It should be noted that according to the assumption that the intra-link is a static AWGN channel, the instantaneous fading channel gain were ignored.

\section{Competing interests}

The authors declare that they have no completing interests.

\section{Acknowledgements}

This research was supported in part by the Japan Society for the Promotion of Science (JSPS) Grant under the Scientific Research KIBAN, (B) No. 2360170 and (C) No. 2256037

\section{Author details \\ ${ }^{1}$ School of Information Science, Japan Advanced Institute of Science and Technology (JAIST), 1-1 Asahidai, Nomi, Ishikawa 923-1292, Japan. Centre for Wireless Communications, University of Oulu, P.O. Box 4500, 90014, Oulu, Finland.}

Received: 1 June 2012 Accepted: 1 February 2013

Published: 23 February 2013

\section{References}

1. M Dohler, Y Li, Cooperative Communications: Hardware, Channel and PHY. (John Wiley and Sons Ltd, West Sussex, 2010)

2. C Edward, VD Meulen, Three-terminal communication channels. Adv. Appl. Prob. 3, 120-154 (1971)

3. G Kramer, M Gastpar, P Gupta, Cooperative strategies and capacity theorems for relay networks. IEEE Trans. Inf. Theory. 51(9), 3037-3063 (2005)

4. Q Chen, in 2010 IEEE Wireless Communications and Networking Conference (WCNC). Energy efficiency analysis in amplify-and-forward and decode-and-forward cooperative networks, (Sydney, 2010), pp. 1-6

5. H Ruiyuan, J Li, in 2005 IEEE 6th Workshop on Signal Processing Advances in Wireless Communications. Exploiting Slepian-Wolf codes in wireless user cooperation (New York, 2005), pp. 275-279
6. K Anwar, T Matsumoto, Accumulator-assisted distributed turbo codes for relay system exploiting source-relay correlation. IEEE Commun. Lett. 16(7), 1114-1117 (2012)

7. J Garcia-Frias, Y Zhao, Near-Shannon/Slepian-Wolf performance for unknown correlated sources over AWGN channels. IEEE Trans. Commun. 53(4), 555-559 (2005)

8. K Anwar, T Matsumoto, Spatially concatenated codes with turbo equalization for correlated sources. IEEE Trans. Signal Process. 60(10) 5572-5577 (2012)

9. H Ochiai, P Mitran, V Tarokh, in 2004 IEEE 60th Vehicular Technology Conference, 2004. VTC2004-Fall, vol. 7. Design and analysis of collaborative diversity protocols for wireless sensor networks, (Los Angeles, 2004), pp. 4645-4649

10. R Youssef, AG i Amat, Distributed serially concatenated codes for multi-source cooperative relay networks. IEEE Trans. Wirel. Commun. 10 253-263 (2011)

11. D Slepian, JK Wolf, Noiseless coding of correlated information sources. IEEE Trans. Inf. Theory. 19(4), 471-480 (1973)

12. M Cheng, K Anwar, T Matsumoto, in 2012 IEEE Vehicular Technology Conference (VTC Fall). Outage analysis of correlated source transmission in block Rayleigh fading channels (Quebec, 2012), pp. 1-5

13. M Schwartz, WR Bennett, S Stein, Communication systems and techniques. IEEE Commun. Mag. 34(5), 9 (1996)

14. VE Dale, PJ Edwin, R Steven, Calculus. (Pearson, Prentice Hall, 2007)

15. M Cheng, K Anwar, T Matsumoto, in International Conference on Communication Systems (ICCS). On the duality of source and channe correlations: Slepian-Wolf relaying viewpoint (Singapore, 2012), pp. 388-392

16. A Goldsmith, Wireless Communications. (Cambridge University Press Stanford University, US, 2005)

17. M Cheng, A Irawan, K Anwar, T Matsumoto, in Progress In Electromagnetics Research Symposium (PIERS). BICM-ID for relay system allowing intra-link errors and a similarity constellation to ARQ schemes, (Kuala Lumpur, 2012), pp. 281-286

18. S ten Brink, Convergence behavior of iteratively decoded parallel concatenated codes. IEEE Trans. Commun. 49(10), 1727-1737 (2001)

19. B Zhao, MC Valenti, Distributed turbo coded diversity for relay channel. Electron. Lett. 39(10), 786-787 (2003)

20. K Fukawa, D Zhao, A Tolli, T Matsumoto, in 5th International ICST Conference on Communications and Networking in China (CHINACOM). Irregular repetition and single parity check coded BICM-ID using extended mapping 'optimal node degree allocation', (Beijing, 2010), pp. 1-6

21. L Hanzo, TH Liew, BL Yeap, Turbo Coding, Turbo Equalisation and Space-time Coding for Transmission over Fading Channels. (John Wiley \& Sons, West Sussex, 2002)

22. L Bahl, J Cocke, F Jelinek, J Raviv, Optimal decoding of linear codes for minimizing symbol error rate (Corresp.) IEEE Trans. Inf. Theory. 20(2), 284-287 (1974)

23. PS Lu, V Tervo, K Anwar, T Matsumoto, in Vehicular Technology Conference (VTC Spring). Low-complexity strategies for multiple access relaying (Budapest, 2011)

24. L Hanzo, R Maunder, J Wang, LL Yang, Near-Capacity Variable-Length Coding. (John Wiley \& Sons, West Sussex, 2010)

25. M Cheng, X Zhou, K Anwar, T Matsumoto, Simple relay systems with BICM-ID allowing intra-link errors. IEICE. E95-B(12), 3671-3678 (2012)

doi:10.1186/10.1186/1687-6180-2013-34

Cite this article as: Cheng et al.: Outage probability of a relay strategy allowing intra-link errors utilizing Slepian-Wolf theorem. EURASIP Journal on Advances in Signal Processing 2013 2013:34. 